Recepción: 07 / 11 / 2016

Aceptación: 22 / 02 / 2017

Publicación: 15 / 05 / 2017

Ciencias de la salud

Artículo de investigación

\title{
Factores de riesgo cardiovascular en una población laboral
}

\author{
Cardiovascular risk factors in a working population
}

Fatores de risco cardiovascular em uma população activa

\author{
Xiomara Caycedo-Casas ${ }^{\mathrm{T}}$ \\ xiomaracaycedo@hotmail.com \\ Mercy T. Sancan-Moreira ${ }^{\mathrm{II}}$ \\ mercy_sancan@hotmail.com \\ Luis R. Loor-Mera ${ }^{\mathrm{II}}$ \\ 1cdoluis_84@hotmail.com
}

Correspondencia: xiomaracaycedo@hotmail.com

${ }^{` M}$ Magister en Gerencia y Liderazgo Educacional, Profesional en Terapia Ocupacional, Docente de la Universidad Laica Eloy Alfaro de Manabí, Manta, Ecuador.

"Magister en Gerencia Educativa, Diploma Superior en Educación Universitaria por Competencias, Licenciada en la Especialización de Terapia Ocupacional, Tecnólogo Medico Especialización Terapia Ocupacional, Docente de la Universidad Laica Eloy Alfaro de Manabí, Manta, Ecuador.

"'Magister en Gerencia Educativa, Licenciado en la Especialización de Terapia Ocupacional, Docente de la Universidad Laica Eloy Alfaro de Manabí, Manta, Ecuador. 


\title{
Resumen
}

Se realizó un estudio epidemiológico, descriptivo y transversal en 110 trabajadores de la ciudad de Manta, evaluados en la consulta de la clínica ocupacional de la Universidad Laica Eloy Alfaro de Manabí, con la finalidad de identificar los factores de riesgos cardiovasculares y la elaboración de una propuesta de programa de intervención. Las variables de interés figuran: edad, sexo, tabaquismo, dislipidemia, sobrepeso, obesidad, sedentarismo, estrés, diabetes, herencia genética, colesterol. Luego de procesada la información se obtuvo como resultados, en lo que se refiere a los factores de riesgo presentes en nuestra población de estudio, los que más predominaron fueron: la hipertensión arterial, la dislipidemia, sobrepeso, estrés y colesterol. Siendo estos considerados por la literatura, como factores de riesgos importantes para la enfermedad cardiovascular.

Palabras clave: enfermedad cardiovascular; estrategia; factores de riesgo.

\begin{abstract}
An epidemiological, descriptive and cross-sectional study was carried out in 110 workers in the city of Manta, evaluated at the consultation of the occupational clinic of the Universidad Laica Eloy Alfaro de Manabí, in order to identify cardiovascular risk factors and The preparation of a proposal for an intervention program. The variables of interest include: age, sex, smoking, dyslipidemia, overweight, obesity, sedentary lifestyle, stress, diabetes, genetic inheritance, cholesterol. After processing the information was obtained as results, with regard to the risk factors present in our study population, the most prevalent were: hypertension, dyslipidemia, overweight, stress and cholesterol. These are considered by the literature as important risk factors for cardiovascular disease.
\end{abstract}

Keywords: cardiovascular disease; strategy; risk factor's.

\section{Resumo}

Um estudo epidemiológico, descritivo transversal foi realizado em 110 trabalhadores na cidade de Manta, avaliado na clínica ocupacional Lay Universidade Eloy Alfaro de Manabi, a fim de identificar fatores de risco cardiovascular e o desenvolvimento de um programa de intervenção proposto. As variáveis de interesse incluem: idade, sexo, tabagismo, dislipidemia, sobrepeso, obesidade, sedentarismo, estresse, diabetes, herança genética, colesterol. Após o processamento da informação obtida como resultado, no que respeita aos factores de risco presentes na nossa 
população de estudo, a maior parte predominante foram: hipertensão, dislipidemia, obesidade, stress e colesterol. Estes sendo considerado pela literatura como importantes factores de risco para doença cardiovascular.

Palavras chave: doença cardiovascular; estratégia; fatores de risco.

\section{Introducción}

Las Enfermedades Cardiovasculares (ECV), son un grupo heterogéneo de enfermedades que afectan tanto al sistema circulatorio como al corazón, de ahí se deriva su nombre (cardiovascular), entre las cuales podemos mencionar a: arteriosclerosis, angina de pecho, hipertensión arterial, hipercolesterolemia, Infarto Agudo de Miocardio (IAM), insuficiencias cardiacas, enfermedad cerebrovascular, trombosis arterial periférica. ${ }^{1}$

Las Enfermedades Cardiovasculares (ECV), son actualmente la mayor causa de muerte, en el mundo. Ellas fueron responsables por más de 17 millones de muertes en 2008, de las cuales tres millones ocurrieron antes de los 60 años de edad, y gran parte podría haber sido evitada. La organización mundial de la salud, estima que en 2030 casi 23,6 millones de personas murieron de enfermedades cardiovasculares. ${ }^{2,3}$

Una de las regiones con mayor riesgo de afectación es Asia Meridional, donde se prevé que la tasa de mortalidad por enfermedades cardiovasculares aumente de 3.8 millones por año en el 2008 a 5.6 millones por año en el $2030 .^{4}$

La enfermedad cerebro vascular aparece con mayor frecuencia en la población de edad avanzada.

Las consecuencias pueden tener diferentes síntomas y gravedad e incluir desde problemas de visión, hasta dificultad para coordinar los movimientos. Esta patología es una de las principales causas de invalidez grave y prolongada en los adultos. ${ }^{5}$

Los siguientes factores le ponen en alto riesgo de sufrir enfermedades cardiovasculares. Es importante saber que algunos de estos factores de riesgo, no tienen síntomas: historia familiar, presión arterial, colesterol elevado, diabetes, fumar tabaco, sobrepeso, sedentarismo, alcoholismo. $^{6}$

En el Ecuador la vigilancia epidemiológica, es un proceso de investigación continúa para la acción y reacción inmediata, que permite profundizar el conocimiento para poder evaluar y tomar 
medidas de intervención, sobre la base del comportamiento de la situación epidemiológica y de sus determinantes de riesgos sociales, ecológicos y biológicos, a través del análisis de indicadores de resultados e impacto. Además, su uso en la administración, permite establecer prioridades para la racionalización de los recursos y mejorar la coordinación de los niveles de prestación de servicios de salud. La vigilancia epidemiológica por lo tanto, es un componente esencial del sistema de salud. Los procesos establecidos en el ministerio de salud pública, obligan a cambios trascendentales en la dinámica de las acciones técnicas desplegadas, en ese sentido la vigilancia epidemiológica, se encuentra en un franco proceso de análisis, cambio y tecnificación informatizada, cuyo resultado final será que los eventos sujetos a vigilancia epidemiológica, estarán definidos bajo parámetros epidemiológicos que permitirán contribuir en forma sustancial en el control de los problemas de salud. ${ }^{7}$

En el orden de las ideas anteriores en el Ecuador, más que una transición epidemiológica se evidencia una acumulación epidemiológica, en la que las enfermedades carenciales y transmisibles comparten espacio con las crónico-degenerativas y los problemas de salud colectiva. Entre las primeras predominan las infecciones respiratorias agudas, las enfermedades diarreicas, la malaria, la tuberculosis pulmonar, las enfermedades inmunoprevenibles, la desnutrición global, la desnutrición crónica y la anemia. Entre las segundas están las enfermedades cerebro vascular, las cardiopatías isquémicas, la hipertensión arterial, la diabetes mellitus, los tumores malignos, el sida y los problemas de salud mental. Este perfil epidemiológico está relacionado con una serie de determinantes tales como los niveles de pobreza y de inequidad en el país. ${ }^{8}$

Según infamo el diario El Comercio 9 en marzo de 2016, la Organización Panamericana de la Salud (OPS), llevó a cabo un estudio en Ecuador sobre las poblaciones en riesgo de padecer enfermedades cardiovasculares. La encuesta recogió datos de 2.231 personas entre 18 y 69 años.

Los resultados fueron arrasadores: un total del 30\% de la población adulta, entre 40 y 69 años corre riesgo de padecer alguna enfermedad, asociada con el mal funcionamiento del sistema cardíaco.

La creencia a la susceptibilidad de padecer una enfermedad, es un paso previo para que las personas inicien un cambio de actitudes frente a los factores de riesgo y eso pueda concluir en un 
cambio de conducta. Sin embargo, una encuesta realizada en toda Europa revela que sólo el 13\% de los encuestados se consideran susceptibles de padecer ECV..$^{10,4}$

La mejor manera de involucrar a una persona, es informarla sobre sus factores de riesgo de ECV de manera clara y didáctica, de tal forma que se despierte el interés y la motivación que le conduzca a cambiar su comportamiento. Cuando esto se acompaña de apoyo por parte del equipo de salud, la posibilidad de reducir el riesgo aumenta considerablemente. ${ }^{11,4}$

La alta prevalencia de Factores de Riesgo Vascular (FRV) en la población, está obligando a poner en marcha programas preventivos, de lo que se considera como una auténtica epidemia de enfermedades cardiovasculares. A este respecto, hay consenso sobre la necesidad de iniciar la prevención de la arteriosclerosis en etapas tempranas de la vida, especialmente en lo que se refiere al fomento de hábitos de vida más saludables. Por todo ello, los reconocimientos médicos anuales que realizan las mutuas de accidentes de trabajo y enfermedades profesionales de la seguridad social a los trabajadores que tienen concertados, constituyen una oportunidad óptima de contribuir a una prevención cardiovascular precoz y eficiente. ${ }^{12}$

En medicina del trabajo y en salud laboral en general, concurren dos circunstancias en estrecha relación con la enfermedad cardiovascular: de una parte lo que ha supuesto el análisis de las condiciones de trabajo, respecto a la historia natural de la enfermedad y de otra, el hecho de que el ámbito laboral ha contribuido particularmente al mejor conocimiento de esta patología habida, cuenta de que en él se han desarrollado numerosos estudios epidemiológicos. ${ }^{13}$

\section{Metodología}

Se realizó un estudio epidemiológico, descriptivo y transversal en 110 trabajadores del centro de trabajo en la cuidad de Manta, evaluados en la consulta de clínica ocupacional de la Universidad Laica Eloy Alfaro de Manabí, con la finalidad de identificar los factores de riesgo cardiovasculares y elaboración de una propuesta, programa de intervención.

Las variables de interés (edad, sexo, tabaquismo, dislipidemia, sobrepeso, obesidad, sedentarismo, estrés, diabetes, herencia genética, colesterol).

Para evaluar la presencia de aglomeración de los factores de riesgos, fue utilizado un puntaje que varió de ninguno (0) a cinco (5) o más factores de riesgo. Fue considerado: ninguno - cuando no 
hubo la exposición a los factores; 1 - cuando hubo exposición a un factor; 2 - exposición a dos factores; 3 - exposición a tres factores; 4 - exposición a cuatro factores; y 5 o más - cuando hubo la exposición a cinco o más factores de riesgo.

El instrumento utilizado fue la encuesta.

La información se obtuvo de la historia clínica laboral. Para procesar los datos se utilizó el paquete estadístico SPSS, versión 11.5 a través del cual se confeccionaron las tablas. Se empleó el porcentaje como medida de resumen.

\section{Resultados}

Tabla 1 Distribución de pacientes por edad y sexo.

\begin{tabular}{|l|c|c|c|c|c|c|}
\hline \multirow{2}{*}{$\begin{array}{l}\text { Grupo de } \\
\text { edad }\end{array}$} & \multicolumn{2}{|c|}{ Masculino } & \multicolumn{2}{c|}{ Femenino } & \multicolumn{2}{c|}{ Total } \\
\cline { 2 - 7 } & No & $\%$ & No & $\%$ & No & $\%$ \\
\hline $30-39$ & 40 & 57.1 & 18 & 45 & 58 & 52.7 \\
\hline $40-49$ & 15 & 21.4 & 13 & 32.5 & 28 & 25.4 \\
\hline $50-59$ & 10 & 14.2 & 8 & 20 & 18 & 16.3 \\
\hline 60 & 5 & 7.1 & 1 & 2.1 & 6 & 5.4 \\
\hline Total & 70 & 63.6 & 40 & 36.3 & 110 & 100 \\
\hline
\end{tabular}

En la serie (tabla 1), predominaron el sexo masculino (63.6\%) y el grupo etario de años 30-39 (58 pacientes, para 52.7\%); edades que también fueron más frecuentes en ambos sexos.

Cuando se realiza el análisis según escolaridad, predominó el nivel universitario, 58 (52.7\%), seguido por técnicos 48 (43.6\%), otros servicios 4. Al tener en cuenta las variables laborales, se pudo apreciar que los trabajadores tenían una media de experiencia laboral de 13.4 años y en el puesto de trabajo de 5,6 años. 
Tabla 2. Factores de riesgo cardiovasculares.

\begin{tabular}{|l|c|c|}
\hline Factores de riesgo & No & $\%$ \\
\hline Hipertensión Arterial & 70 & 63.6 \\
\hline Sobrepeso & 62 & 56.3 \\
\hline Obesidad & 22 & 20 \\
\hline Tabaquismo & 30 & 27.2 \\
\hline Sedentarismo & 10 & 9.0 \\
\hline Dislipidemia & 64 & 58.1 \\
\hline Diabetes & 32 & 29.0 \\
\hline Herencia genética & 16 & 14.5 \\
\hline Estrés & 60 & 54.5 \\
\hline Colesterol & 50 & 45.4 \\
\hline
\end{tabular}

Entre los factores de riesgos preponderantes al realizarle la historia clínica (tabla 2), figuraron en ese orden: hipertensión arterial (63.6\%), dislipidemia (58.1\%), sobrepeso (56.3\%), estrés (54.5\%), le continuo el colesterol (45.4\%) también aparecieron otras, pero con menor frecuencia: diabetes, tabaquismo, obesidad, herencia genética y el sedentarismo.

En la casuística se halló predominio de los pacientes del sexo masculino, con presencia de factores de riesgo, la hipertensión arterial presento el mayor por ciento (80.0\%), en el sexo femenino fue de 14 (20.0\%), con respecto a los demás factores de riesgo su comportamiento siempre fue mayor en el sexo masculino.

Se verifica que $38.8 \%$ de los individuos estudiados de 50 a 59 años, poseen cinco o más factores de riesgo cardiovascular. Con edad de 30 a 39 años, el 25\% no posee factores de riesgo cardiovasculares. En la edad de los 40 a los 49 años, 9 pacientes $(33,33 \%)$ presentaron aglomeración de dos factores de riesgo.

\section{Discusión}

El estudio puso de manifiesto la desigual distribución de los factores de riesgo cardiovascular y la elevada prevalencia de alguno de ellos, en un grupo poblacional de edad media relativamente joven y mayoritariamente masculino. La escasa proporción de mujeres en la población objeto del estudio limita la posibilidad de extraer conclusiones válidas y fiables por sexos. 
En un reciente meta-análisis con datos de 18 estudios de cohortes que incluía a más de 250.000 sujetos seguidos hasta 50 años, se puso de manifiesto que, en ausencia de tabaquismo, diabetes mellitus, con concentraciones de colesterol inferiores a $180 \mathrm{mg} / \mathrm{dL}$ y cifras de tensión arterial inferiores a 120/80 $\mathrm{mmHg}$, la enfermedad cardiovascular es prácticamente inexistente antes de los 85 años de edad. ${ }^{14}$

En este estudio un factor de riesgo importante fue la hipertensión arterial, predominando en el sexo masculino. Resultados que coinciden con estudio realizado por Santana López et al., ${ }^{15}$. Se estima que la hipertensión ha causado 9,4 millones de fallecimientos y el 7\% de la carga de morbilidad; si no se controla, la hipertensión es causa de accidentes cerebrovasculares, infarto de miocardio, insuficiencia cardiaca, demencia, insuficiencia renal y ceguera.

Prácticamente todas las naciones, la prevención y el control de la HAS ocasiona implicaciones importantes y la utilización de nuevas estrategias y abordajes que identifiquen con más precisión los individuos en situación de riesgo, ofrecen beneficios tanto para el individuo con hipertensión, como para la sociedad. Sin embargo, por ser una enfermedad crónica, el control de la HAS requiere acompañamiento y tratamiento durante toda la vida, en el que participan medidas farmacológicas y no farmacológicas. ${ }^{3}$

Hay sólidas pruebas científicas de los beneficios para la salud, que reporta la reducción de la presión arterial mediante intervenciones poblacionales e individuales; la prevalencia mundial de HTA en adultos mayores de 18 años al cierre del año 2014 fue del 22\%, según datos de la OMS. $^{15}$

La hipercolesterolemia es uno de los factores de riesgo cardiovascular más sensible a las medidas de prevención y control. Entre los diversos factores implicados en las enfermedades cardiovasculares, la hipercolesterolemia, y principalmente los valores elevados de colesterol ligado a proteínas de baja densidad (c-LDL), se consideran factores mayores o causales, y las estrategias dirigidas a lograr su control son las más eficaces. El estudio Multiple Risk Factor Intervention Trial (MRFIT), demostró una relación continua y gradual (sin umbral para su comienzo) entre la colesterinemia y la mortalidad total y por cardiopatía isquémica. Además, reducir la colesterinemia disminuye la incidencia y la mortalidad por cardiopatía isquémica y la enfermedad cardiovascular en general, tanto en prevención primaria como secundaria. ${ }^{16}$ 
Otros autores ${ }^{4}$ en investigación realizada acerca de riesgo cardiovascular en la población laboral. Impacto en aspectos preventivos, obtuvieron en la serie que el sobrepeso muestra relaciones estadísticas significativas con riesgo incrementado de hipertensión arterial, glucemia basal y diabetes, incremento de grasa corporal, visceral y perímetro de cintura, menor masa muscular, índices elevados de lípidos, estilos de vida no saludables y coexistencia con patologías de riesgo cardiovascular. La actuación preventiva desde las empresas parte de un conocimiento previo de la situación de riesgo cardiovascular de los trabajadores, facilitando una planificación más eficaz y menor coste de las acciones en promoción de la salud e intervención coordinadas con los especialistas involucrados en su control y seguimiento.

La hipertensión arterial, dislipidemia, sobrepeso, estrés y el colesterol predominaron como factores de riesgo, como se aprecia en esta investigación.

A pesar de que el tabaquismo, la diabetes, el sedentarismo y la obesidad mostraron por ciento menores con el resto de los factores de riesgo estudiados en la investigación, se le deben de tener la misma atención. Según Santana $\mathrm{S}$ et al., ${ }^{15}$ evidencias científicas demuestran la importancia del control del peso para evitar la ocurrencia de HTA, cardiopatía isquémica, enfermedades cerebro vasculares, diabetes mellitus, síndrome metabólico y algunos tipos de cáncer. ${ }^{18}$

En el marco de las observaciones anteriores, según Molina ${ }^{17}$, la diabetes mellitus se ha convertido en una pandemia y con ello en un problema de salud pública; el paciente diabético tiene dos a cuatro veces mayor riesgo de enfermedad coronaria y ataque cerebrovascular que él no diabético.

La OMS, plantea que de las seis regiones, la de las Américas es la más afectada por la obesidad; se estimó que para el cierre del año 2015 alrededor de 300 millones de personas estarían afectadas por esta enfermedad (39\% de la población total). ${ }^{15}$

El hábito de fumar tiene consecuencias para la salud, el entorno y la economía del individuo. El nivel de exposición al humo afecta tanto a los consumidores como a aquellos que son forzados a inhalar el humo del cigarro ambiental. Molina ${ }^{17}$, notifica que el riesgo de enfermedad cardiovascular comienza a disminuir desde el momento en que se deja de fumar; hay 50\% de reducción de eventos cardiovasculares e infarto no fatal y hacia el tercer año el riesgo disminuye prácticamente al de los que nunca han fumado. En una investigación realizada en Japón ${ }^{3}$ se 
observó que el tabaquismo y HAS son los dos principales factores de riesgo para la mortalidad de adultos por enfermedades no transmisibles.

Para dar por concluido, en lo que se refiere a los factores de riesgo, presentes en la población de estudio los más prevalentes fueron: la hipertensión arterial, la dislipidemia, sobrepeso, estrés y colesterol siendo estos considerados por la literatura como factores de riesgo importantes para la enfermedad cardiovascular.

\section{Propuesta de programa de intervención}

- Objetivo: confeccionar un programa de intervención educativo, tomando como base los resultados de la investigación.

\section{- Actividades:}

- capacitar a los profesionales de la salud en el manejo de la enfermedad cardiovascular y sus factores de riesgos.

- Promover hábitos de vida saludables y factores protectores en población expuesta o no a factores de riesgo.

- Estimular el auto cuidado en el paciente y la participación de la familia durante este proceso.

- Favorecer la accesibilidad y adherencia de los pacientes al programa.

- Estas intervenciones deben ser conducidas de modo a permitir que los individuos discutan asuntos referentes a su condición de salud y los factores de riesgo que participan y, al mismo tiempo, sean estimulados.

- Formas de organización: cursos, talleres, seminarios, entrenamientos, debates y otras técnicas de educación para la salud útil, para el logro del objetivo.

- Evaluación: seguimiento y control de las intervenciones formuladas dentro del programa, contribuyendo a la disminución del riesgo en estos individuos.

- Resultados esperados: detectar individuos diagnosticados y aquellos asintomáticos con factores que puedan generar riesgo de enfermar y sufrir de eventos cardiovasculares, a través de diferentes 
vías y estrategias de captación, con el objetivo de realizar un plan terapéutico adecuado de acuerdo a los factores de riesgo identificados.

Estas son las intervenciones más costo-eficaces identificadas para reducir las enfermedades cardiovasculares y se encuentran al alcance de las comunidades si se les educa oportunamente y se comprometen con su salud.

\section{Referencias Bibliográficas}

1- Lo que la población debe saber sobre las enfermedades cardiovasculares. 2016 [citado 8 julio 2016]. Disponible en: pifrecv.utalca.cl/docs/int_publico/ecv_pag_web.pdf

2- World Health Organization (WHO). Global atlas on cardiovascular disease prevention and control. Mendis S, Puska P, Norrving B editors. Geneva: world health organization; 2011

3- Radovanovic CAT, Santos LA, Carvalho MDB, Marcon SS. Hipertensión arterial y otros factores de riesgo asociados a las enfermedades cardiovasculares en adultos Rev. Latino-Am. Enfermagem. 2014 [citado 30 agosto 2016]; 22(4):547-53. Disponible en: DOI: 10.1590/01041169.3345.2450 www.eerp.usp.br/rlae

4- Vicente-Herrero Ma. Teófila, Terradillos García Ma. Jesús, Capdevila García Luisa M, Ramírez Iñiguez de la Torre Ma. Victoria, López-González Ángel Arturo. Riesgo cardiovascular en la población laboral: impacto en aspectos preventivos. Rev. Mex. Cardiol. 2014 [citado 17 junio 2016]; 25(2): 73-81. Disponible en: http://www.scielo.org.mx/scielo.php?script=sci_arttext\&pid=S0188$21982014000200002 \& \operatorname{lng}=\mathrm{es}$.

5- Factores de riesgo cardiovascular 2016 [citado 8 julio 2016]. Disponible en:http://www.cuidateplus.com/enfermedades/enfermedades-vasculares-y-del-corazon/factoresde-riesgo-cardiovascular.html

6- Enfermedades cardiovasculares. 2016 [citado 30 de agosto 2016]. Disponible en: http://www.brighamandwomens.org/online/enespanol/default.aspx 
7- Epidemiologia. Vigilancia epidemiológica. Gobierno Nacional de la república del Ecuador. 2016 [citado julio 2016]. Disponible en: instituciones.msp.gob.ec/dps/cotopaxi/index.php?option=com_content\&view=article\&id=13\&Ite $\operatorname{mid}=44$

8- Perfil de sistema de salud: Ecuador, monitoreo y análisis de los procesos de cambio y reforma Washington, D.C.: OPS, 2008. ISBN 978-92-75-33267-2

9- Las enfermedades cardiovasculares son la primera causa de muerte en Ecuador. Diario El Comercio 2016 [citado 8 julio 2016]. Disponible en: http://www.elcomercio.com/tendencias/enfermedadescardiovasculares-muertes-ecuador-cifrasjuangabriel.html

10- Erhardt L, Hobbs FD. Public perception of cardiovascular risk in five European countries the react survey. Int J Clin Pract. 2002; 56 (9): 638-644

11- Benner JS, Erhardt L, Flammer M, Moller RA, Rajicic N, Changela K et al. A novel programme to evaluate and communicate 10-year risk of CHD reduces predicted risk and improvepatient's modifiable risk factor profile. Int J Clin Pract. 2008; 62 (10): 1484-1498

12- Sánchez-Chaparro M A, Román-García J, Calvo-Bonacho E, Gómez-Larios T, FernándezMeseguer A, Sáinz-Gutiérrez J, Cabrera-Sierra M, Á García-García A, Rueda-Vicente J, GálvezMoraleda A, González-Quintela A . Prevalencia de factores de riesgo vascular en la población laboral española. Rev Esp Cardiol. 2006 [citado 30 agosto 2016]; 59:421-30 - 59 (05). Disponible en: DOI: $10.1157 / 13087894$

13- C. Ulldemolins Salvador; C. Armelles Reig, F Juárez Doyague, A. Casinello Apoita; A. Harto Castaño. Factores de riesgo cardiovascular en la población laboral española. Sección técnica [citado 12 junio 2016]. 5-2000, páginas 11 a 23. Disponible en: www.insht.es/InshtWeb/Contenidos/Documentacion/.../seccionTecTextCompl2.pdf

14- Berry JD, Dyer A, Cai X, Arside DB, Ning H, Tho-mas A, et al. Life time risks of cardiovascular dis-ease. N Engl J Med. 2012; 366:321-9 
15- Santana S, Perdomo MC, Oramas A, González A. Factores de riesgo cardiovasculares y turno de trabajo en una población laboral. Revista Cubana de Salud y Trabajo 2016; 17(4):42-5

16- Escribano Hernández Alfonso, Vega Alonso Agustín Tomás, Lozano Alonso José Eugenio, Álamo Sanz Rufino, Castrodeza Sanz José Javier, Lleras Muñoz Siro. Dislipidemias y riesgo cardiovascular en la población adulta de Castilla y León. Gac Sanit. 2010 [citado 12 junio 2016]; 24(4): 282-287. Disponible en: http://scielo.isciii.es/scielo.php?script=sci_arttext\&pid=S0213-91112010000400004\&lng=es

17- Molina de Salazar Dora I. Propuesta en prevención del riesgo cardiovascular. Rev. Col. Cardiol. 2008 [citado 12 junio 2016]; 15(5): 203-206. Disponible en: http://www.scielo.org.co/scielo.php?script=sci_arttext\&pid=S0120$56332008000500001 \& \operatorname{lng}=\mathrm{en}$ 